\title{
Experimental Study on the Inhibitory Effect of HMME-PDT on the Cariogenic Bacteria in Dental Plaque Biofilms of Rats
}

\author{
Xue Liu ${ }^{1}$, Yadong Tao ${ }^{2}$, Xuehai Wang ${ }^{1}$, Meihui Yang ${ }^{1}$, Hongjie Guo ${ }^{1}$, Feng Huo ${ }^{2,}$ * \\ ${ }^{1}$ University Health Service, Chengde Medical University, Chengde, China \\ ${ }^{2}$ Department of Dental, The Affiliated Hospital of Chengde Medical University, Chengde, China
}

Email address:

siyu5652022@163.com (Xue Liu),434364845@qq.com(Feng Huo)

${ }^{*}$ Corresponding author

\section{To cite this article:}

Xue Liu, Yadong Tao, Xuehai Wang, Meihui Yang, Hongjie Guo, Feng Huo. Experimental Study on the Inhibitory Effect of HMME-PDT on the Cariogenic Bacteria in Dental Plaque Biofilms of Rats. European Journal of Clinical and Biomedical Sciences.

Vol. 5, No. 5, 2019, pp. 66-72. doi: 10.11648/j.ejcbs.20190505.11

Received: September 23, 2019; Accepted: November 4, 2019; Published: November 25, 2019

\begin{abstract}
Object: To investigate the inhibitory effect of HMME-PDT on the cariogenic bacteria we designed different photosensitizer concentration and different lighting time. Therefore, we determined the optimal photosensitizer parameters and time parameters. Besides we explored the advantages of HMME-PDT in inhibiting the cariogenic bacteria in dental plaque biofilms of rats. We provided theoretical and experi- mental basis for the application of PDT for dental caries prevention. Method: The caries model was constructed with Wistar rats. We grouped the rats in accordance with the principle of randomization. We used the above two methods to evaluate the inhibitory effect of HMME-PDT on the cariogenic bacteria in dental plaque biofilms of rats. Results: 1. The bacteriostatic effect of HMME-PDT on bacterial plaque in rat oral cavity was studied in different concentration of photosensitizer. The tablet colony count results showed with the increase of concentration of photosensitizer stress the bacteria inhibitive rate was increased. Compared to the negative control group and positive control group, group E1 changed most obviously. 2. The bacteriostatic effect of HMME-PDT on bacterial plaque in rat oral cavity was studied in different exposure time. The tablet colony count results showed with the increase of exposure time the bacteria inhibitive rate was increased. Compared to the negative control group and positive control group, group E2 changed most obviously. 3. Results of the calcium ion dissolution detected by atomic absorption spectrophotometer showed with the increase of exposure time the calcium ion dissolution was increased in each group in 48 hours. Compared to the negative control group, the calcium ion dissolution of each group was decreased significantly $(\mathrm{P}<0.05)$. Compared to the positive control group, the calcium ion dissolution of each group was decreased significantly $(\mathrm{P}<0.05)$. Conclusion: Photodynamic therapy can effectively inhibit the production of caries in the biofilm of rats and reduce calcium ion solubility in early caries.
\end{abstract}

Keywords: Photodynamic Therapy, Dental Caries, Plaque Biofilm

\section{Introduction}

Caries is a disease that the dental hard tissue occurred chronic progressive destruction mainly caused bacteria. bacterial infection is the direct cause of caries. The occurrence and development of caries can only be mediated by dental plaque. The occurrence and development of caries can only be mediated by dental plaque. The formation process of dental plaque biofilm changes dynamically in time and space. It is a place where bacteria live and communicate signals between cells [1]. The intact structure of plaque biofilm has strong resistance and tolerance to host defense system and decreased sensitivity to antibiotics. Therefore, through appropriate methods to affect the growth and metabolism of bacteria in plaque biofilm and to interfere the formation of plaque or promote the dissociation of plaque, the purpose of preventing caries can be achieved. Photodynamic therapy (PDT) is a new type of therapy that utilizing light to activate photosensitizers and produce photochemical effects on inhibiting pathogenic microorganisms. It can effectively treat bacterial diseases without developing resistance. Due to its unique advantages in bacteriostatic, it has attracted more and more attention from scholars at home and abroad [2]. 
PDT, also known as photoactivation disinfection technology, is a new antimicrobial method [3]. The basic elements of PDT include photosensitizer, light source and oxygen. They worked together. Domestic and foreign literatures have discussed the effect of PDT on in vitro mixed bacteria plaque biofilm [4]. However, the effects of photodynamic therapy with 532 semiconductor laser as light source and porphyrin monomethyl ether as photosensitizer on the bacteriostatic of mixed oral plaque biofilm in rats are rarely discussed. Hematology monomethyl (HMME) is a new type of photosensitive agent independently developed in China. Its chemical composition is stable, and it has the advantages of short time of avoiding light, high yield of single weight oxygen, long wavelength of absorption, low relative content of porphyrin causing phototoxic reactions in normal tissues. HMME has been used in the clinical treatment of some diseases. In this study, the inhibition effect of HMME-PDT on oral mixed bacteria plaque biofilm in rats was completed by optimizing and selecting appropriate PDT parameters.

In this study, we mainly studied the inhibitory effect of $532 \mathrm{~nm}$ semiconductor laser on oral mixed bacterial plaque biofilm in rats under different photosensitizer concentrations and different illumination times. In this study, we mainly studied the inhibitory effect of 532nm semiconductor laser on oral mixed bacterial plaque biofilm in rats under different photosensitizer concentrations and different illumination times. It provides theoretical and experimental basis for the application of HMME-PDT in the clinical practice of caries prevention.

\section{Method and Materials}

\subsection{Materials}

\subsubsection{Bacterial Strain}

Streptococcus mutants (S. mutants UA159), streptococcus sanguis (S. sangius ATCC10556), actobacillus acidophilus (L. acidophilus ATCC4356), Actinomyces viscosus (A. viscosus ATCC19246).

\subsubsection{Reagent}

Photosensitizer: hematoporphyrin monomethyl ether (Fudan Zhanjiang biomedicine co., LTD., Shanghai), dissolved into a final concentration of $10 \mathrm{mg} / \mathrm{ml}$ and stored at $4^{\circ} \mathrm{C}$ in dark.

TPY liquid medium: $4.5 \mathrm{~g}$ peptone, $1.2 \mathrm{~g}$ yeast extract, $0.6 \mathrm{~g}$ $\mathrm{Na} 2 \mathrm{CO} 3,0.6 \mathrm{~g} \mathrm{NaCl}, 3 \mathrm{~g}$ glucose, $0.6 \mathrm{~g}$ K2HPO4, $1.8 \mathrm{~g}$ $\mathrm{KH} 2 \mathrm{PO} 4$, distilled water added to $300 \mathrm{ml}$, mixed with the above ingredients, autoclaved and stored at $4{ }^{\circ} \mathrm{C}$ for later use.

\subsubsection{Laboratory Apparatus}

Semiconductor laser (wavelength: 630nm, institute of biomedical engineering, Chinese academy of medical sciences)

Purification table (vs-1300u, Suzhou purification equipment factory) Electric thermostatic incubator (hh-bll.360, Tianjin experimental instrument factory)
Monobioc electronic balance (mettler Toledo weighing equipment co., LTD.)

Vortex oscillator (eppendorf ag, Germany)

Low-speed centrifuge (ld52b, Beijing),

Anaerobic incubator (THERMD),

Optical microscope.

\subsection{Method}

\subsubsection{Recovery and Growth of Bacteria}

The four experimental strains revived for $48 \mathrm{~h}$ were inoculated in the TPY liquid culture medium, respectively, and incubated in $37^{\circ} \mathrm{C}$ anaerobic environment $\left(\mathrm{CO}_{2} 80 \%, \mathrm{~N}_{2} 10 \%\right.$, $\mathrm{H}_{2} 10 \%$ ) for $24 \mathrm{~h}$. After the smear examination showed pure culture, the concentration of bacterial liquid was adjusted to $1 \times 108 \mathrm{CFU} / \mathrm{mL}$ for later use.

\subsubsection{Establishment of Caries Induced Model}

A total of 96 Wistar rats were chose which weaned 21 days after birth at the same litter and age. Sampling of young mice was randomly selected to observe the colonization of the original flora in the oral cavity of young mice before taking antibiotics. During the period of $22-24$ days, mice were exposed to antibiotics drinking water with $1 \mathrm{~g} / \mathrm{kg}$ of body weight $(200 \mathrm{mg} / \mathrm{ml}$ penicillin, $1500 \mathrm{mg} / \mathrm{L}$ streptomycin), and mice were sampled at 25 days to detect the inhibition of oral bacteria. Mixed bacteria solution of four kinds of bacteria were added to the dental surfaces of rats at a ratio of $1: 1$ for 3 consecutive days from 25 to $27 \mathrm{~d}$ at the age of rats. A $2000 \#$ caria-causing feed containing $1 \%$ mixed bacteria drinking water was given. The bacterial colonization was detected by random sampling at the age of 27 days.

\subsubsection{Group 96 Rats Were Randomly Divided into 12 Groups}

Group A was normal saline negative treatment group. Group B was the positive control group of sodium fluoride. Other groups, depending on the concentration of photosensitizer, are divided into C1 group $20 \mathrm{mg} / \mathrm{L}$, D1 group $30 \mathrm{mg} / \mathrm{L}$, E1 group $40 \mathrm{mg} / \mathrm{L}, \mathrm{F} 1$ group $50 \mathrm{mg} / \mathrm{L}$ and $\mathrm{G} 1$ group $60 \mathrm{mg} / \mathrm{L}$. After incubation in dark for $5 \mathrm{~min}$, a semiconductor laser with a wavelength of $532 \mathrm{~nm}$ is exposed to $90 \mathrm{~s}$, with a speckle radius of $0.5 \mathrm{~cm}$ and a power density of $0.14 \mathrm{w} / \mathrm{cm} 2$. According to different exposure time, it is divided into $\mathrm{C} 2$ group 30s, D2 group 60s, E2 group 90s, F2 group 120s, G2 group $150 \mathrm{~s}$. After $5 \mathrm{~min}$ incubation with a photosensitive concentration of $40 \mathrm{mg} / \mathrm{L}$, the semiconductor laser with a wavelength of $532 \mathrm{~nm}$ was irradiated with a spot radius of $0.5 \mathrm{~cm}$ and a power density of $0.14 \mathrm{w} / \mathrm{cm} 2$. Each group consisted of 8 rats.

\subsubsection{Experimental Method}

At the age of 28 days, the rats were treated with PDT according to the above groups. The rats' teeth were coated with photosensitizer and incubated for $5 \mathrm{~min}$. After irradiation, the same experimenter swabbed the teeth on the maxillary surfaces in a clockwise direction. The cotton swab was put into a centrifuge tube containing $5 \mathrm{~mL}$ bacterial culture solution according to different groups. They were vibrated for 
$2 \mathrm{~min}$ at $3000 \mathrm{r} / \mathrm{min}$, and then continuously diluted. The suspension was coated on the TSA culture plate at $37^{\circ} \mathrm{C}$ and incubated in a constant temperature anaerobic incubator for 48h. Then observe the results. The rats were treated once a week according to the above methods, and the test results were executed until the end of the $56 \mathrm{~d}$ experiment.

\subsubsection{Effect of Photodynamic Therapy on Calcium Ion Dissolution in Caries}

After the rats were killed, the teeth of each isolated group were treated with $1 \mathrm{ml}$ normal saline, which was vibrated by a vibrator for $3 \mathrm{~min}$, and $6 \mathrm{ml}$ fresh culture solution was added. The culture solution was taken for $1 \mathrm{ml}$ at 6 time points of $8 \mathrm{~h}$, $16 \mathrm{~h}, 24 \mathrm{~h}, 32 \mathrm{~h}, 40 \mathrm{~h}$ and $48 \mathrm{~h}$ respectively. The content of calcium ions was measured by atomic absorption spectrophotometer. The measurement method was atomic absorption spectrometry with the resonance line of $422.7 \mathrm{~nm}$. The absorbance of calcium standard solution was firstly used to draw the standard curve. The absorbance of culture medium was determined. The calcium ion concentration in culture medium was converted by the standard curve method. Under constant experimental conditions, the absorbance and the concentration of calcium ions in solution conform to lambert-beer's law $\mathrm{A}=\mathrm{KC}, \mathrm{A}$ is the absorbance, and $\mathrm{C}$ is the sample concentration [5].

\subsection{Statistical Approach}

The results were expressed as mean \pm standard deviation $( \pm \mathrm{s})$. SPSS 2.0 was used to analyze the data. After normal test, all the values of each group were in line with normal, and the rank-sum test was used to compare the differences, which were statistically significant $(\mathrm{P}<0.05)$.

Colony count compared with repetitive measure analysis of variance, treatment group of pairwise comparisons between the Student - Newman - Keuls to test. $\mathrm{P}<0.05$ was considered to be statistically significant.

\section{Result}

\subsection{Influence of PDT to the Cariogenic Bacteria Colony Amount in Dental Plaque Biofilms}

We established caries model of rats. And then we obtained samples from the teeth of rats at different time points and inoculated them to the media. After inoculation for $72 \mathrm{~h}$ we counted the number of colony and recorded (Table 1). We also performed a bar graph to intuitively demonstrate the colony amount in different groups and time points (Figure 1).

Table 1. Comparison of the bacteria in dental plaque biofilms between experimental groups. $(n=8, \bar{x} \pm s, C F U / m L)$.

\begin{tabular}{lllll}
\hline Group & First week & Second week & Third week & Fourth week \\
\hline A & $2.915 \pm 0.357$ & $3.092 \pm 0.192$ & $3.111 \pm 0.235$ & $3.693 \pm 0.212$ \\
B & $2.736 \pm 0.279$ & $2.894 \pm 0.381$ & $2.963 \pm 0.213$ & $3.502 \pm 0.100^{*}$ \\
C1 & $2.489 \pm 0.371$ & $2.920 \pm 0.325$ & $2.943 \pm 0.202$ & $2.752 \pm 0.132^{*}$ \\
D1 & $2.512 \pm 0.342$ & $2.851 \pm 0.203$ & $2.893 \pm 0.169$ & $2.706 \pm 0.110^{*}$ \\
E1 & $2.552 \pm 0.238$ & $2.785 \pm 0.268$ & $2.845 \pm 0.159$ & $2.005 \pm 0.141^{*} \Delta$ \\
F1 & $2.561 \pm 0.295$ & $2.726 \pm 0.256$ & $2.851 \pm 0.139$ & $2.051 \pm 0.122^{*} \Delta$ \\
G1 & $2.559 \pm 0.295$ & $2.695 \pm 0.188$ & $2.844 \pm 0.133$ & $2.132 \pm 0.146^{*}$ \\
F & 1.906 & 2.025 & 2.240 & $1.732 \pm 0.10 .125^{*} \Delta$ \\
\hline
\end{tabular}

${ }^{*}$ Compared to Group saline-treated group, there was significant difference $(P<0.05)$

${ }^{\triangle}$ Compared to Group NaF group, there was significant difference $(P<0.05)$

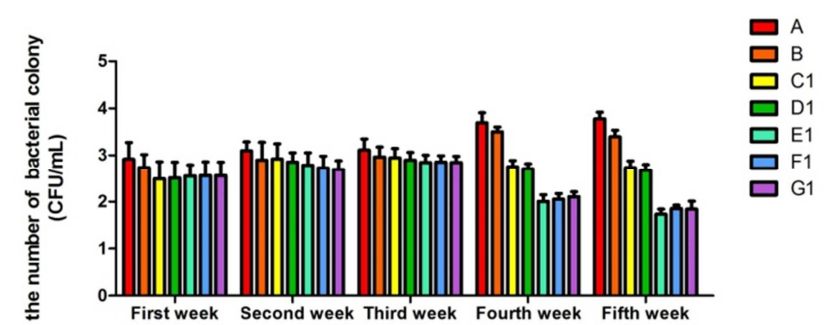

Figure 1. The number of bacterial colonies in different groups and time points.

The results showed that the time factor alone had a statistically significant effect on the growth of mixed bacteria in the rat mouth $(\mathrm{F}=26.618, \mathrm{P}<0.05)$. The treatment factor had a statistically significant effect on the growth of mixed bacteria in the rat mouth $(\mathrm{F}=112.691, \mathrm{P}<0.05)$, and there was an interactive effect between the treatment and the time factor ( $\mathrm{F}$ interaction $=21.442, \mathrm{P}<0.05)$. There was no significant difference in the number of bacterial colonies in the first, second and third weeks $(\mathrm{P}>0.05)$. There was no significant difference in the number of bacterial colonies in the fourth and fifth weeks. In the fourth and fifth weeks, Group C1, D1, E1, F1 and G1 were significantly different from Group A and B. The difference between E1 group (40mg/L group) and sodium fluoride group was the most obvious. However, as the concentration increased again, there was no significant difference between group E1 and group F1 and G1. Thus, the best E1 group (40mg/L group) was obtained.

\subsection{Effect of Photodynamic Therapy on Calcium ion Dissolution in Caries}

After the rats were killed, the teeth of each isolated group were treated with $1 \mathrm{ml}$ normal saline, which was vibrated by a vibrator for $3 \mathrm{~min}$, and $6 \mathrm{ml}$ fresh culture solution was added. The culture solution was taken for $1 \mathrm{ml}$ at 6 time points of $8 \mathrm{~h}$, $16 \mathrm{~h}, 24 \mathrm{~h}, 32 \mathrm{~h}, 40 \mathrm{~h}$ and $48 \mathrm{~h}$ respectively (Table 2). We performed line chart to display the calcium ion dissolution directly (Figure 2). 
Table 2. The calcium ion dissolution at any time point after treatment $\left(n=10, \quad \bar{x}_{ \pm s, u g / m L)}\right.$.

\begin{tabular}{lllllll}
\hline Group & $\mathbf{8 h}$ & $\mathbf{1 6 h}$ & $\mathbf{2 4 h}$ & $\mathbf{3 2 h}$ & $\mathbf{4 0 h}$ & $\mathbf{4 8 h}$ \\
\hline A & $2.126 \pm 0.160$ & $5.274 \pm 0.190$ & $7.180 \pm 0.210$ & $9.186 \pm 0.206$ & $16.847 \pm 0.257$ & $20.408 \pm 0.201$ \\
B & $1.120 \pm 0.181$ & $2.159 \pm 0.146$ & $3.415 \pm 0.272$ & $4.360 \pm 0.121$ & $5.430 \pm 0.148$ & $7.480 \pm 0.242$ \\
C1 & $1.098 \pm 0.103$ & $2.104 \pm 0.100$ & $2.914 \pm 0.215$ & $3.101 \pm 0.204$ & $4.349 \pm 0.192$ & $6.048 \pm 0.202$ \\
D1 & $0.623 \pm 0.159$ & $0.956 \pm 0.131$ & $1.501 \pm 0.155$ & $2.512 \pm 0.132$ & $3.114 \pm 0.129$ & $5.144 \pm 0.133$ \\
E1 & $0.000 \pm 0.000^{*} \Delta$ & $0.199 \pm 0.078^{*} \Delta$ & $0.554 \pm 0.147^{*} \Delta$ & $0.765 \pm 0.207^{*} \Delta$ & $0.927 \pm 0.137^{*} \Delta$ & $1.457 \pm 0.132^{*} \Delta$ \\
F1 & $0.000 \pm 0.000^{*} \Delta$ & $0.251 \pm 0.091^{*} \Delta$ & $0.992 \pm 0.174$ & $1.344 \pm 0.259$ & $1.800 \pm 0.261$ & $2.876 \pm 0.316$ \\
G1 & $0.103 \pm 0.033^{*} \Delta$ & $0.361 \pm 0.083$ & $1.232 \pm 0.259$ & $1.542 \pm 0.195$ & $1.948 \pm 0.172$ & $2.971 \pm 0.316$ \\
F value & $454.082^{*}$ & $2180.395^{*}$ & $1198.351^{*}$ & $2199.127^{*}$ & $8182.658^{*}$ & $7707.675^{*}$ \\
\hline
\end{tabular}

*Compared to Group saline-treated group, there was significant difference $(P<0.05)$

${ }^{\triangle}$ Compared to Group NaF group, there was significant difference $(P<0.05)$

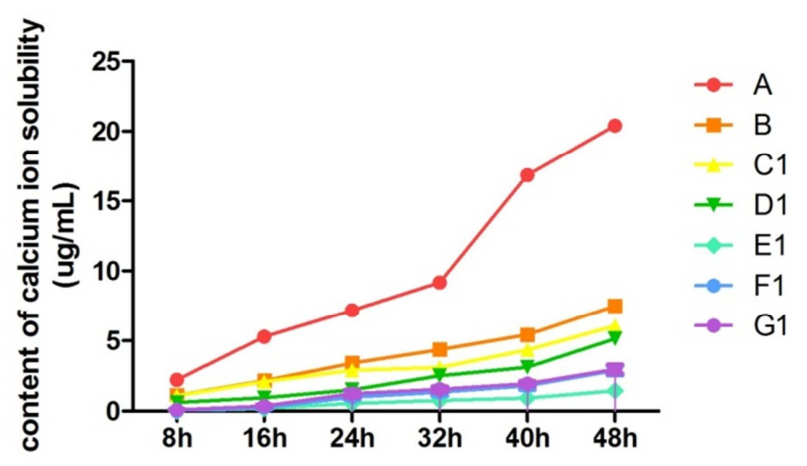

Figure 2. The calcium ion dissolution at any time point after treatment.

As shown in the figure, the amount of calcium ions dissolved in each group increased with the extension of time within 48 hours. As shown in the table, compared with the normal saline negative control group, sodium fluoride and each time group can reduce the amount of calcium ions dissolved at each time point. Compared with the sodium fluoride group, the amount of calcium ions dissolved in each group decreased at each time point. Among them, the dissolution amount of calcium ions in E1 group $(40 \mathrm{mg} / \mathrm{L})$ at each time point was significantly lower than that in sodium fluoride group $(\mathrm{P}<0.05)$.

\subsection{Influence of PDT to the Cariogenic Bacteria Colony Amount in Dental Plaque Biofilms}

We established caries model of rats. And then we obtained samples from the teeth of rats at different time points and inoculated them to the media. After inoculation for $72 \mathrm{~h}$ we counted the number of colony and recorded (Table 3 ). We also performed a bar graph to intuitively demonstrate the colony amount in different groups and time points (Figure 3).

Table 3. Comparison of the bacteria in dental plaque biofilms between experimental groups. $(n=8, \bar{x} \pm s, C F U / m L)$.

\begin{tabular}{lllll}
\hline Group & First week & Second week & Third week & Fourth week \\
\hline A & $2.915 \pm 0.357$ & $3.092 \pm 0.192$ & $3.111 \pm 0.235$ & $3.693 \pm 0.212$ \\
B & $2.736 \pm 0.279$ & $2.894 \pm 0.381$ & $2.963 \pm 0.213$ & $3.502 \pm 0.100^{*}$ \\
C2 & $2.638 \pm 0.255$ & $2.870 \pm 0.281$ & $2.909 \pm 0.117$ & $2.739 \pm 0.163^{*}$ \\
D2 & $2.705 \pm 0.225$ & $2.832 \pm 0.229$ & $2.878 \pm 0.186$ & $3.394 \pm 0.140^{*}$ \\
E2 & $2.636 \pm 0.221$ & $2.781 \pm 0.119$ & $2.902 \pm 0.102$ & $2.721 \pm 0.127^{*}$ \\
F2 & $2.603 \pm 0.249$ & $2.805 \pm 0.277$ & $2.874 \pm 0.111$ & $2.015 \pm 0.225^{*} \Delta$ \\
G2 & $2.656 \pm 0.204$ & $2.722 \pm 0.159$ & $2.890 \pm 0.067$ & $1.797 \pm 0.149^{*} \Delta$ \\
F value & $1.318^{*}$ & $1.824^{*}$ & $2.240^{*}$ & $2.082 \pm 0.127^{*} \Delta$ \\
\hline
\end{tabular}

${ }^{*}$ Compared to Group saline-treated group, there was significant difference $(P<0.05)$

${ }^{\triangle}$ Compared to Group NaF group, there was significant difference $(P<0.05)$

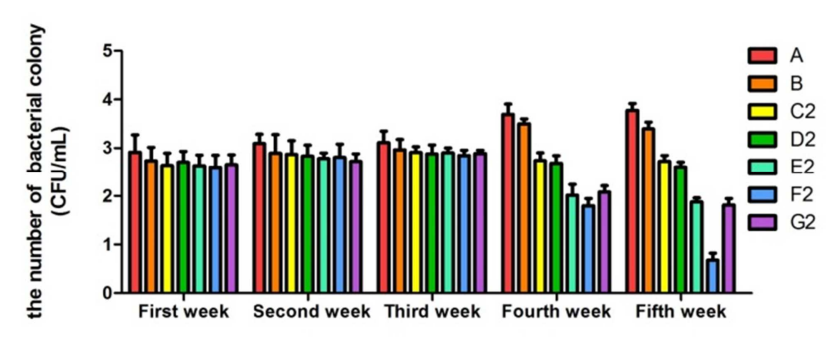

Figure 3. The number of bacterial colonies in different groups and time points.

The results showed that the time factor alone had a statistically significant effect on the growth of mixed bacteria in rats $(\mathrm{F}=34.162, \mathrm{P}<0.05)$. The treatment factor had a statistically significant effect on the growth of mixed bacteria in rats $(\mathrm{F}=134.239, \mathrm{P}<0.05)$. The treatment and time factor had an interactive effect ( $\mathrm{F}$ interaction $=29.408, \mathrm{P}<0.05$ ). There was no significant difference in the number of colonies in the first, second and third weeks $(\mathrm{P}>0.05)$. There was no significant difference in the number of colonies in the fourth and fifth weeks. In fourth and fifth week Group C2, D2, E2, F2, G2 were significantly different from $\mathrm{A}$ and $\mathrm{B}$. The difference of E2 group, normal saline group and sodium fluoride group is the most obvious. As the concentration increases, there is no significant difference between E2, F2 and G2 groups. Thus, the best E2 group (90s group) is obtained. 


\subsection{Effect of Photodynamic Therapy on Calcium Ion Dissolution in Caries}

After the rats of Group 2 were killed, the teeth of each isolated group were treated with $1 \mathrm{ml}$ normal saline, which was vibrated by a vibrator for $3 \mathrm{~min}$, and $6 \mathrm{ml}$ fresh culture solution was added. The culture solution was taken for $1 \mathrm{ml}$ at 6 time points of $8 \mathrm{~h}, 16 \mathrm{~h}, 24 \mathrm{~h}, 32 \mathrm{~h}, 40 \mathrm{~h}$ and $48 \mathrm{~h}$ respectively (Table 4 ). We performed line chart to display the calcium ion dissolution directly (Figure 4).

Table 4. The calcium ion dissolution at any time point after treatment $(n=10, \bar{x} \pm s, u g / m L)$.

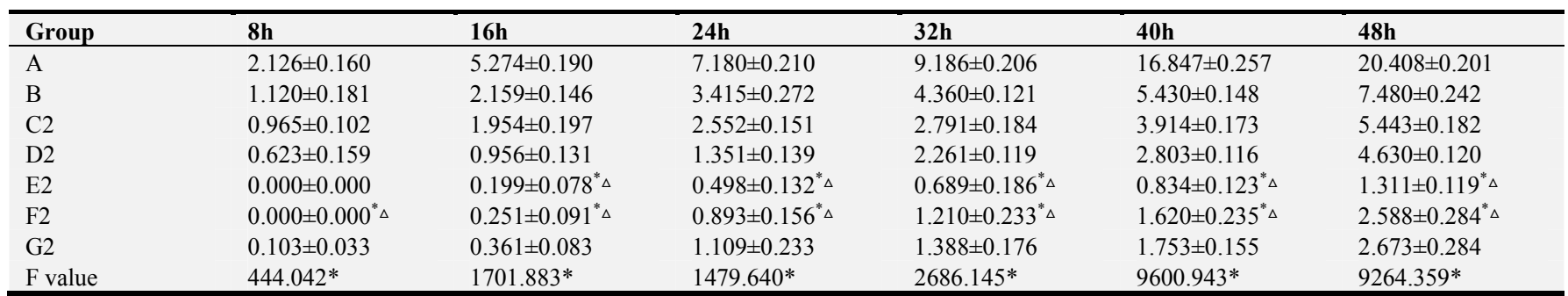

*Compared to Group saline-treated group, there was significant difference $(P<0.05)$

${ }^{\triangle}$ Compared to Group NaF group, there was significant difference $(P<0.05)$

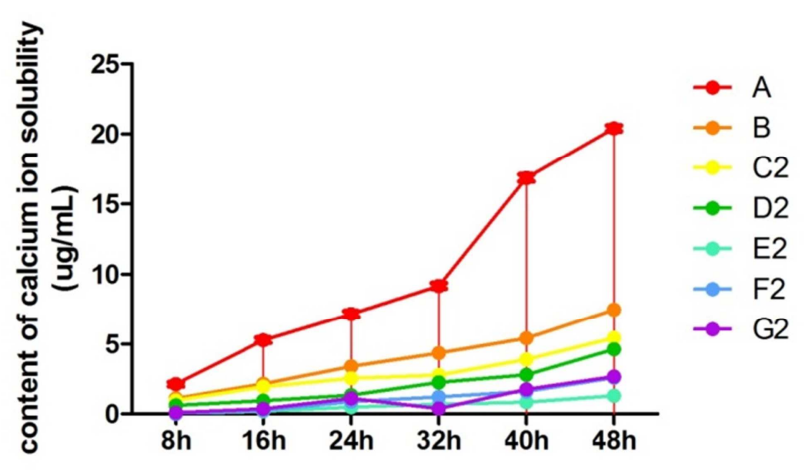

Figure 4. The calcium ion dissolution at any time point after treatment.

As shown in the figure, the amount of calcium ions dissolved in each group increased with the extension of time within 48 hours. As shown in the table, compared with the normal saline negative control group, sodium fluoride and other groups can reduce the amount of calcium ions dissolved at each time point. Compared with the sodium fluoride group, the amount of calcium ions dissolved decreased. The calcium ion dissolution of E2 group (90s group) is significantly less than that of sodium fluoride group $(\mathrm{P}<0.05)$.

\section{Discussion}

At present, antibiotics are widely used in the prevention and treatment of caries, but the following problems exist. Firstly, it is difficult to reach effective concentration of antibiotics due to the influence of oral saliva and gingival crevicular fluid. Secondly, it can develop drug resistance in bacteria that cause caries. Last but not Least, it may destroy normal flora and lead to conditioned pathogenic infection [6]. As for the defects of antibiotics in preventing caries, the researchers hope to find a new and effective method to prevent caries. Target cells were stimulated by light at appropriate wavelength. Then they producted reactive oxygen species (ROS) which can attack cell structure through oxidation. The process was named photodynamic therapy (PDT).
Such damage may be the oxidative damage of cell membrane or protein, and when the accumulation of oxidative damage exceeds a certain threshold, cells begin to die [7]. Photosensitizer is the core substance of PDT. The photosensitizer HMME used in this experiment has the advantages of high purity (98\%), high single-state oxygen quantum yield, short light-avoiding period and good tissue selectivity [8]. Studies have shown that PDT to accompany actinobacillus [8], oral biofilm to caries streptococcus mutants [9] and [10] in enterococcus of mixed bacteria plaque biofilm has obvious killing effect, but most of the previous related research limitations in vitro experiments, animal model study, or a single bacteria and PDT in animal models of the influence of the mixed bacteria plaque biofilm coverage is still rare. In this study, a rat caries model was established to study the effect of PDT on plaque biofilm of four major caries pathogenic bacteria. The results show that the first 3 weeks after PDT treatment, on the molar surface of the rat there was no statistically significant difference in living bacterium amount between saline group and sodium fluoride group. Until four weeks after PDT treatment the living bacterium amount of Group E2 (HMME $40 \mathrm{mg} / \mathrm{L}$, exposure time 90 seconds) was less than that of normal saline group and sodium fluoride. It can be inferred that the decrease of bacteria amount in the mixed bacteria plaque on the molars of rats was related to the frequency of PDT.

The reduction of bacteria amount on rat molar surface is associated with PDT treatment times. The number of colonies decreased in different degrees along with the increasing of PDT treatment times. In the fourth week of the experiment, the bacteria number on the molar surface of rats in the 90s group with 40mg/L HMME-PDT treatment time was the least. It was significantly lower than that of other treatment groups. It is indicated that the concentration of photosensitizer and the time of laser irradiation were important factors affecting the PDT effect. Under the same incubation time, the bactericidal effect is more obvious in the $40 \mathrm{mg} / \mathrm{L}$ HMME-PDT group. The bactericidal effect is more obvious in the 90s laser irradiation group under the same photosensitizer concentration. So the best parameter of the bactericidal effect of HMME-PDT on 
the mixed bacteria plaque biofilm of rat molar surface is the $40 \mathrm{mg} / \mathrm{L}$ photosensitizer and 90s laser irradiation time.

Tooth decay occurs, bacteria caused caries produced acid in the metabolic process which can change the local microenvironment. What's more inorganic compounds was dissolved and damaged. Calcium and phosphorus was removed out of the teeth. The mechanical strength of the tooth body was decreased. It has created favorable conditions for caries bacteria invasion. Therefore precipitation of calcium, phosphorus ion can be indirectly speed up the development of dental decay process [11]. In this study, calcium ion dissolution method was used to analyze the progress of caries. Atomic absorption spectrometry was used to detect the trace changes of calcium ion concentration, which has high sensitivity and can meet the requirements of this study. The experimental results showed that within 48 hours, the number of ions dissolved in each group increased with the extension of time. The amount of calcium ions dissolved in each group changed. PDT may denature collagen and non-collagen proteins in teeth, change the spiral spacing, and form sites between collagen that are easy to bind calcium ions $[12,13$, 14]. The amount of calcium ions dissolved in PDT group decreased at each time point, which had significantly difference from that in normal saline group and sodium fluoride group. It is indicated that PDT could reduce the amount of calcium ions dissolved in early caries, not only preventing the continued demineralization of enamel, but also promoting the re-mineralization of enamel. Therefore, PDT has a broad clinical application prospect in the prevention of caries.

Our results show that PDT can effectively inhibit the growth of mixed bacteria in the oral cavity of rats and play a role in preventing caries. This experiment confirmed that the semiconductor laser with the wavelength of $532 \mathrm{~nm}$, at the concentration of HMME $40 \mathrm{mg} / \mathrm{L}$, and the laser irradiation time of $90 \mathrm{~s}$, is the best parameter for inhibiting the mixed bacteria plaque biofilm of HMME-PDT on the molar surface of rats. In addition, this study only explored the inhibitory effect of HMME-PDT on bacteria, but the effects on the teeth, pulp, periodontal soft and hard tissues of rats were not elaborated. Therefore, this is the focus of future in-depth research, providing theoretical and experimental basis for the clinical application of HMME-PDT to prevent caries.

\section{Conclusion}

HMME-PDT has a significant inhibitory effect on the cariogenic bacteria in dental plaque biofilms of rats. The experiments prove that the optimal photosensitizer concentration of HMME-PDT was $40 \mathrm{mg} / \mathrm{L}$ and the optimal time of exposure duration was 90s. Photodynamic therapy can effectively inhibit the production of caries in the biofilm of rats and reduce calcium ion solubility in early caries. PDT not only prevents the enamel from demineralization, but also promotes the remineralization of enamel. HMME-PDT has a broad clinical application prospect in the prevention of caries.

\section{Recommendations}

Author Contributions These authors contributed equally to this work.

\section{Conflict of Interest Statement}

The authors declare no conflict of interest.

\section{Acknowledgements}

This study received support from Chengde science and technology research and development program (Project Nos. 201606A043), Youth fund project of education department of Hebei province (Project Nos. QN2019079) and Natural science foundation project of Chengde medical college (Project Nos. 201722).

\section{References}

[1] Auschill TM, Hein N, Hellwing E, et al, Effect of two antimicrobial agentson early in situ bilfilm formation [J]. Clin Periofontol, 2005, 32 (2): 147-152.

[2] Lilburn TG, Gu J, Cai H, et al. Comparative genomics of the family Vibrionaceae reveals the wide distribution of genesencod in virulence-associated proteins $[\mathrm{J}]$. BMC Genomics, 2010, 10 (11): 369-372.

[3] Souza L, Brito P, Oliveira J, et al. Photodynamic therapy with two different photosensitizers as a supplement to instrumentation irrigation procedures in promoting intracanal reduction of Enterococcus faecalis. [J] Endod, 2010, 36 (2): 292-296.

[4] Xiu ming Chang, Zhao hui Zou, Hui juan Yin. Effect of photodynamic therapy on dental plaque biofilm cariogenic bacteria and its mechanism [J]. Journal of Oral Science Research. 2012, 28 (4): 303-309.

[5] Hussein dental AS, Ghasheer HF, Ramli NM, et al. Salivary trace elements carves magroup of mufti-ethnic schoolchildren in relation to Shah Alam, Malaysia [J]. Eur J Paediatr Dent. 2013; 14 (2): 113-118.

[6] Michael Wilson. Lethal photosensitization of oral bacteriaan ditsp-tential application in the photodynamic therapy of oral in fections [J]. Photochem PhotobiolSci, 2004, 3 (5): 412-418.

[7] Lin hua Ge, Rong shu. Application of photodynamic therapy in the treatment of periodontal disease $[\mathrm{J}]$. Chinese Journal of Conservative Dentistry, 2007, 17 (2): 113-116.

[8] Qiu tao Fu, Ying Gu, Fan guang Liu, etal. Observation on photosensitive effect of serum methyl ether in vitro $[\mathrm{J}]$. Acta Laser Biology Sinica, 2000, 9 (2): 137-141.

[9] Goulart RD, Bolean M, Paulino TD, et al. Photodynamic Therapy in Plank tonican Biofilm Cultures of Aggregati bacteraction mycetem-comitans [J]. Photomed LaserSurg, 2010, 28 (Supp11): 53-60.

[10] Zou ZH, Gao P, Yin HJ, et al. Investigation of photodynamic therapy on strep to coccusmutans of oral biofilm [J]. Chinese Optics Letters, 2008, 6 (12): 947-949. 
[11] Fonseca MB, Tessare PO, Pallota RC, et al. Photodynamic therapy for root canals infected with Enterococcusfaecalis [J]. Photomed La- serSurg, 2008, 26 (3): 209-213.

[12] Salmon C R, Tomazela D M, Ruiz K G, et al. Proteomic analysis of human dental cementum and alveolar bone [J]. J Proteomics. 2013; 8 (9): 544-555.
[13] Nissan J, Assif D, Gross MD, et al. Effect of low intensity laser irradiation on surgically created bony defects in rats $[\mathrm{J}]$. Oral Rehabil, 2006, 33 (8): 619-624.

[14] Khadra M, Kasem N, Haanaes HR, et al. Enhancement of bone formation in ratcalvarial bone defects using low-level laser therapy [J]: Oral Surg Oral Med Oral Pathol Oral Radiol Endod, 2004, 97 (6): 693-700. 